\title{
Diagnostic Utility of Third-Look, Contrast-Enhanced Sonography Followed by Needle Biopsy for MRI, But Not Second-look Ultrasonography-detected Breast Lesions
}

\author{
TOMOHIRO MIYAKE, SEUNG JIN KIM, MASAFUMI SHIMODA, NAOFUMI KAGARA, \\ TOMONORI TANEI, YASUTO NAOI, KENZO SHIMAZU and SHINZABURO NOGUCHI
}

\author{
Department of Breast and Endocrine Surgery, Osaka University Graduate School of Medicine, Osaka, Japan
}

\begin{abstract}
Background/Aim: To clarify the diagnostic utility of third-look, contrast-enhanced ultrasonography (CEUS) for MRI-detected breast lesions undetectable by unenhanced, second-look ultrasonography (MRI+/USlesions). Patients and Methods: Clinical stage 0-IIA breast cancer patients who underwent CEUS for incidental MRI+/US- lesions ( $n=27$; cohort 1$)$ and patients with breast lesions detected only by MRI, to be examined by CEUS ( $n=15$; cohort 2$)$, were retrospectively analyzed. Results: Of the 42 MRI+/US- lesions, 23 (55\%) were detected by CEUS and then examined by needle biopsy. Pathological examination showed that 10 of these 23 lesions were malignant. None of the 19 lesions undetected by CEUS were found to be histologically malignant or developed malignancy with a median follow-up of 18.5 months. The accuracy, sensitivity, and specificity of CEUS plus needle biopsy were $98 \%, 91 \%$, and 100\%, respectively. Conclusion: Third-look CEUS followed by needle biopsy is useful in the initial diagnosis of MRI+/US-lesions.
\end{abstract}

Contrast-enhanced magnetic resonance imaging (MRI) of the breast is a useful tool for precise evaluation of a spreading tumor and plays an important role in the preoperative planning of breast-tissue resection margins. It is reported that preoperative bilateral breast MRI examination helps reveal contralateral breast cancer undetectable by other modalities such as mammography and ultrasonography (US) and

Correspondence to: Seung Jin Kim, Department of Breast and Endocrine Surgery, Osaka University Graduate School of Medicine, 22-E10 Yamada-oka, Suita City, Osaka 565-0871, Japan. Tel: +81 0668793772, Fax: +81 0668793779, e-mail: kimsj@onsurg.med.osakau.ac.jp

Key Words: Breast cancer, magnetic resonance imaging (MRI), contrast-enhanced ultrasonography (CEUS), needle biopsy, diagnostic utility. contributes to the decrease of contralateral breast cancer recurrence (1), but whether MRI decreases ipsilateral breast cancer recurrence after breast-conserving surgery is controversial $(2,3)$.

Conventionally, it is pointed out that breast MRI shows a high sensitivity and a low specificity (4). Lesions detected by breast MRI are usually examined by secondlook US, with various detection rates (5). Frequency of malignancy of "probably benign lesions" detected by breast MRI (BI-RADS category 3) is reported to be very low but not zero (6). At present, there is no consensus about the management of such lesions, that is, MRI-guided biopsy or regular follow-up with MRI, when such lesions are undetectable by second-look US. On the other hand, MRIguided biopsy is usually indicated for MRI-detected breast lesions, "suspicious for malignancy" (BI-RADS category 4), and "highly suggestive of malignancy" (BI-RADS category 5) $(7,8)$. The European Society of Breast Imaging (EUSOBI) guidelines recommend that MRI should be offered by the institutions where MRI-guided biopsy is available or by those that closely connect with such institutions (9). However, MRI-guided biopsy is expensive and time consuming, and thus available only in a limited number of institutes in Japan.

Recently, new methods, such as real-time virtual sonography and contrast-enhanced US (CEUS), using SonoVue ${ }^{\circledR}$ (Bracco S.p.A., Milan, Italy), applied to a limited number of patients $(n=10)$ have been reported to be useful for the diagnosis of suspicious lesions detected by breast MRI $(10,11)$. CEUS, using perflubutane microbubble (Sonazoid $^{\circledR}$; Daiichi-Sankyo, Tokyo, Japan), with a mean diameter of $2-3 \mu \mathrm{m}$ is reported to demonstrate a superiority over unenhanced US in terms of differential diagnosis of breast lesions, with no severe adverse events (12). In the present study, the diagnostic utility of CEUS was retrospectively investigated by Sonazoid ${ }^{\circledR}$ and CEUS-guided needle biopsy for breast MRI lesions undetectable by unenhanced second-look US. 


\section{Patients and Methods}

Patients. Patients with clinical stage 0-IIA primary breast cancer who underwent third-look, contrast-enhanced US (CEUS) for MRIdetected incidental lesions (BI-RADS category 3-5) which were not detected by unenhanced, second-look US (MRI+/US- lesions) $(\mathrm{n}=27$; cohort 1$)$ and patients with only MRI+/US- breast lesions who underwent third-look CEUS for the index lesions (BI-RADS category 3-4) $(\mathrm{n}=15$; cohort 2) between June 2015 and October 2017 at Osaka University Hospital were retrospectively analyzed in this study. Clinical and radiological findings, including age, clinical stage, symptoms associated with MRI+/US- lesions, positional relationship of MRI+/US- lesions with index tumors, and MRI BIRADS classifications are shown in Table I. This retrospective observational study was approved by the institutional review board of Osaka University Hospital (approval number 17299).

Breast MRI examination. All MR images were acquired with a $1.5-\mathrm{T}$ or 3.0-T unit in the prone position using a standard dedicated breast array coil at our hospital $(n=27)$ and the other institutes $(n=15)$. For each examination, T1-weighted dynamic contrast MR images were analyzed for this study, including one unenhanced and three contrast-enhanced sequences after a rapid intravenous bolus injection of a gadolinium-containing contrast agent, using a threedimensional (3D), T1-weighted, fat-saturated gradient echo sequence. MRI-detected lesions were classified into BI-RADS MRI categories (13) by one radiologist at each institution and, in addition, two breast specialists at our hospital.

Contrast-enhanced US and pathological diagnostic procedure. After perflubutane microbubble (Sonazoid ${ }^{\circledR}$; Daiichi-Sankyo, Tokyo, Japan) was injected intravenously, the suspected breast area with the MRI+/US- lesion was examined by CEUS, using Logiq E9 with XDclear ${ }^{\circledR}$ (GE Healthcare, Tokyo, Japan) (14). When the CEUS detected MRI+/US- lesions, the patients underwent needle biopsy such as US-guided, vacuum-assisted biopsy (VAB; $n=21$ ) or fineneedle aspiration cytology (FNAC; $\mathrm{n}=2)$ for pathological diagnosis.

Statistical analysis. Diagnostic accuracy of CEUS alone or CEUS followed by needle biopsy for MRI+/US- lesions was evaluated by the following parameters: accuracy, sensitivity, and specificity. Of the 42 lesions, 20 were surgically resected, and final pathological diagnosis, that is, benign or malignant, was made on the surgical specimens, and 22 were not resected but regularly followed up. If the lesions were followed up for more than 12 months with no evidence of malignancy, they were considered benign in the calculation of diagnostic accuracy (15). Regular follow-up was performed by physical examination at 3-6-month intervals, annual mammography, and by US in at least 6 months intervals with or without breast MRI.

JMP ${ }^{\circledR}$ PRO 14 software (SAS Institute Inc., Cary, NC, USA) was used for all statistical analyses. Associations between the two parameters were analyzed using Fisher's exact test. $p$-Values $<0.05$ were determined to be statistically significant.

\section{Results}

Preoperative diagnosis of MRI-detected lesions. Clinical and radiological findings of the $42 \mathrm{MRI}+\mathrm{US}-$ lesions are shown in Figure 1. CEUS detected 55\% (23/42) of these lesions. Of
Table I. Clinical and radiological findings of patients in cohort 1 and cohort 2.

\begin{tabular}{lcc}
\hline & $\begin{array}{c}\text { Cohort } 1 \\
(\mathrm{n}=27)\end{array}$ & $\begin{array}{c}\text { Cohort 2 } \\
(\mathrm{n}=15)\end{array}$ \\
\hline Age (year) & $50(33-84)$ & $41(34-64)$ \\
$\quad$ Median (range) & 8 & - \\
Clinical stage (main tumor) & 13 & - \\
0 & 6 & - \\
I & & \\
IIA & 25 & 9 \\
Symptom associated & 2 & 5 \\
with MRI lesion & 0 & 1 \\
None & & \\
Abnormal nipple discharge & $(2.0-60.0)$ & $13.0(2.0-45.0)$ \\
Induration & & \\
MRI lesion size (mm) & & - \\
Median (range) & $11(5)$ & - \\
Positional relationship of & 16 & 3 \\
MRI lesion with main tumor & & 12 \\
Ipsilateral (same segment) & 15 & 0 \\
Contralateral & 11 & \\
MRI BI-RADS category & 1 & \\
3 & & \\
4 & & \\
5 & &
\end{tabular}

MRI: Magnetic resonance imaging; BI-RADS: Breast Imaging Reporting and Data System.

Table II. A comparison between pathological diagnoses by needle biopsy and surgical specimen of MRI+/US- lesions.

\begin{tabular}{lcc}
\hline & \multicolumn{2}{c}{ No. of patients } \\
\cline { 2 - 3 } & $\begin{array}{c}\text { Needle } \\
\text { biopsy }\end{array}$ & $\begin{array}{c}\text { Surgical } \\
\text { specimen }\end{array}$ \\
\hline Malignant & & \\
Invasive lobular carcinoma & 1 & 1 \\
Mucinous carcinoma & 1 & 1 \\
DCIS & 7 & 7 \\
FNAC, positive & 1 & 1 mucinous carcinoma \\
Benign & & \\
Intraductal papilloma & 1 & N.R. \\
Usual ductal hyperplasia & 3 & N.R. \\
Flat epithelial atypia & 1 & N.R. \\
Fibrocystic disease & 2 & N.R. \\
Fibroadenomatous lesion & 1 & N.R. \\
Normal breast & 4 & DCIS, 3 N.R. \\
FNAC, negative & 1 & N.R. \\
\hline
\end{tabular}

DCIS: Ductal carcinoma in situ; FNAC: fine needle aspiration cytology; N.R.: not resected.

the 42 lesions, $10(24 \%)$ were diagnosed as malignant preoperatively by VAB $(n=9)$ or FNAC $(n=1)$. Finally, following surgery, 3 of the 10 lesions were diagnosed as 


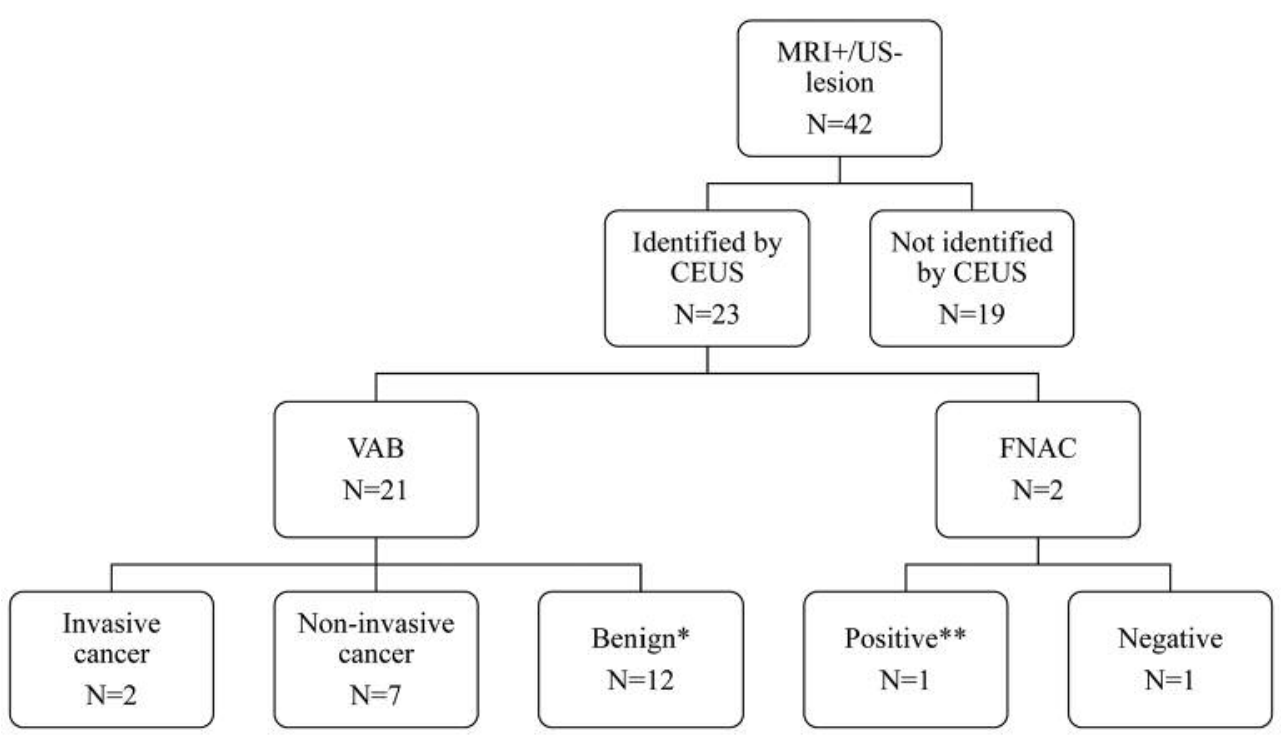

Figure 1. Flow chart of diagnostic process of MRI+/US- lesions by CEUS-guided biopsy. Forty-two MRI+/US- (MRI-detectable/US-undetectable) lesions in 42 patients were included in this study. Of them, 23 lesions were detectable and 19 were undetectable by CEUS. The CEUS-detected lesions received VAB $(n=21)$ or FNAC $(n=2)$. VAB revealed two invasive and seven non-invasive cancers and 12 benign diseases, and $F N A C$ revealed one invasive cancer and one benign disease. *One patient continued bloody nipple discharge after CEUS-guided VAB and then underwent microdochectomy of the lesion, which was finally diagnosed as noninvasive cancer. **One FNAC-positive MRI+/US-lesion turned out to be a mucinous carcinoma based on the pathological examination of the surgical specimen. MRI: Magnetic resonance imaging; CEUS: contrast-enhanced ultrasonography; VAB: vacuum-assisted biopsy; FNAC: fine-needle aspiration cytology.

invasive breast carcinomas and 7 of them as ductal carcinomas in situ (DCIS). Representative cases of MRI+/US- lesions that were detected by third-look CEUS but not by second-look US are shown in Figure 2.

Of the $13 \mathrm{MRI}+/ \mathrm{US}-$ lesions diagnosed as benign by CEUS-guided biopsy, one was finally diagnosed as DCIS by microdochectomy performed due to the continuing bloody nipple discharge after CEUS-guided VAB. With respect to the other $12 \mathrm{MRI}+/ \mathrm{US}-$ lesions, none of them were resected but were followed up for a median of 29.5 months (range $=13-42$ months), without an evidence of malignancy. A comparison between the pathological diagnoses by needle biopsy and surgical specimen of MRI+/US- lesions is shown in Table II.

On the other hand, of the $19 \mathrm{MRI+}$-US- lesions not detected by CEUS, nine were finally diagnosed as benign after surgery, and 10 were not resected but followed up for a median of 18.5 months (range=14-31 months) with no evidence of malignancy.

Positional relationship of MRI+/US-lesion with index tumor in cohort 1 . In cohort 1 , all of the MRI+/US- lesions were identified as incidental lesions by preoperative MRI for primary breast cancer. Positional relationship of MRI lesions with index tumor as well as correlation of preoperative with postoperative pathological diagnosis of these incidental lesions are shown in Table III. Five MRI+/US- lesions were in the same quadrant as the index tumor, and six were in a different quadrant from the index tumor; 16 were in the contralateral breast. All of the MRI+/US- lesions diagnosed as malignant by CEUS-guided needle biopsy proved to be malignant by pathological analysis of the surgical specimens.

Identification rate of MRI+/US- lesions by CEUS and their preoperative pathological diagnosis according to MRI BIRADS category. MRI+/US- lesions included 18 category 3 lesions, 23 category 4 lesions, and one category 5 lesion, according to BI-RADS classification (Table IV). A significantly higher identification rate of the MRI+/USlesions by CEUS was observed in category $4 / 5$ lesions than in category 3 lesions $(79 \%$ vs. $22 \%, p<0.05)$, and final pathological diagnosis as malignant was significantly more frequent in the category $4 / 5$ lesions than in the category 3 lesions ( $42 \%$ vs. $6 \%, p<0.05)$.

Diagnostic accuracy of CEUS alone or CEUS followed by needle biopsy for MRI+/US- lesions. Comparison of CEUS alone and CEUS followed by needle biopsy for MRI+/US- lesions with regard to the final diagnosis is shown in Table V. As for CEUS alone, accuracy, 


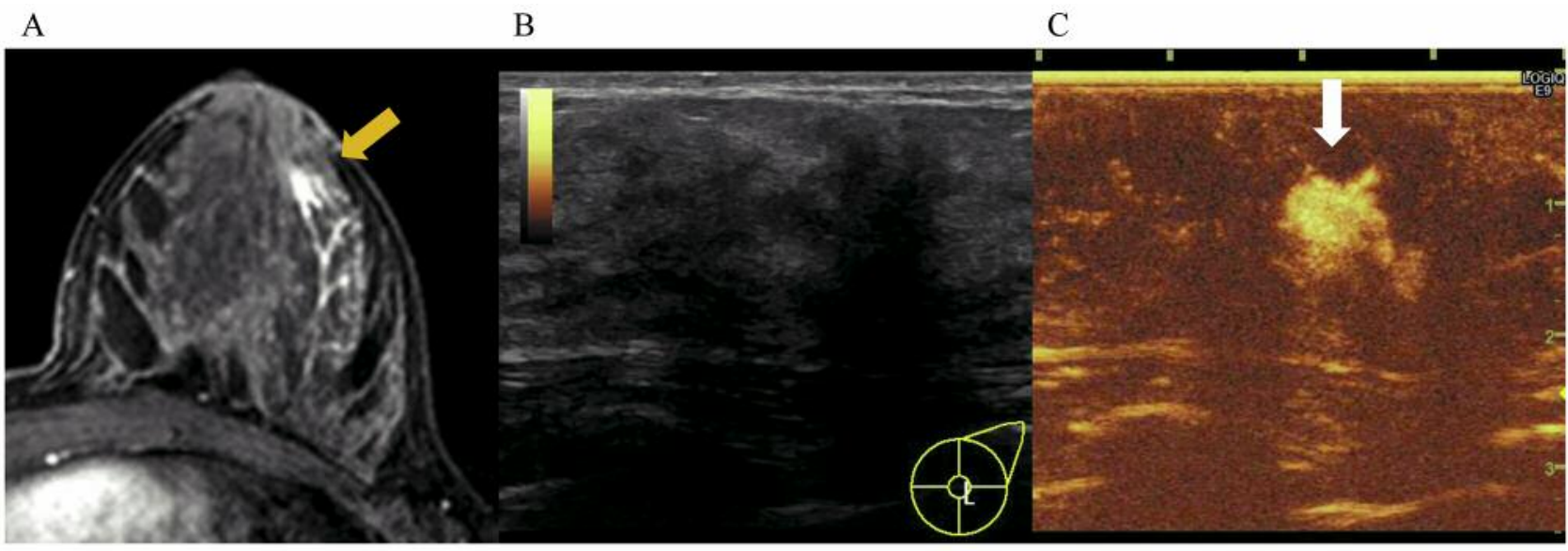

Figure 2. Case presentation. A: Breast contrast-enhanced MRI of a 34-year-old female patient with the symptom of left bloody nipple discharge showed an $8 \mathrm{~mm}$ size mass at a distance of $12 \mathrm{~mm}$ from the left nipple toward the 4 o'clock direction (yellow arrow). Second-look US did not show obvious abnormalities in the suspected area of the MRI+/US-lesion, and third-look CEUS was performed subsequently. B: Paralleled display of third-look CEUS demonstrated that the MRI+/US- lesion was not detected in a normal B-mode setting. C: It was detected clearly as a 6 mm size enhanced mass in a CEUS-mode setting (white arrow). Third-look CEUS-guided VAB revealed that the MRI+/CEUS-lesion was a low-grade DCIS.

Table III. Frequency of patients with malignant tumors in MRI+/USlesions, according to their positional relationship to index tumors in cohort 1 .

\begin{tabular}{lccc}
\hline & $\begin{array}{c}\text { No. of } \\
\text { patients }\end{array}$ & \multicolumn{2}{c}{$\begin{array}{c}\text { No. of patients with } \\
\text { malignant lesions }\end{array}$} \\
\cline { 3 - 4 } & & $\begin{array}{c}\text { Preoperative } \\
\text { diagnosis* }\end{array}$ & $\begin{array}{c}\text { Final } \\
\text { diagnosis }\end{array}$ \\
\hline Ipsilateral & 11 & $3(27)$ & $3(27)$ \\
Same quadrant & 5 & 0 & 0 \\
Different quadrant & 6 & 3 & 3 \\
Contralateral & 16 & $4(25)$ & $5(31)^{* *}$ \\
\hline
\end{tabular}

MRI: Magnetic resonance imaging. *Diagnosis by CEUS-guided core needle biopsy. **One patient with the symptom of bloody nipple discharge was diagnosed as DCIS by microdochectomy, which was performed at the same time as the operation for the index tumor.

Table IV. Identification rate of MRI-detected, but not second-look USdetected lesions by CEUS and their preoperative pathological diagnosis, according to MRI BI-RADS category.

\begin{tabular}{lccc}
\hline $\begin{array}{l}\text { MRI BI-RADS } \\
\text { category }\end{array}$ & $\begin{array}{c}\text { No. of } \\
\text { patients }\end{array}$ & $\begin{array}{c}\text { Identification } \\
\text { rate }(\%)\end{array}$ & $\begin{array}{c}\text { Frequency of } \\
\text { cancer }(\%)\end{array}$ \\
\hline 3 & 18 & $4(22)$ & $0(0)^{*}$ \\
4 & 23 & $18(78)$ & $9(39)$ \\
5 & 1 & $1(100)$ & $1(100)$ \\
\hline
\end{tabular}

MRI: Magnetic resonance imaging; CEUS: contrast-enhanced ultrasonography. *One benign lesion diagnosed by core needle biopsy was later upgraded to DCIS by microdochectomy performed due to continuous bloody nipple discharge.
Table V. Comparison of CEUS alone and CEUS plus core needle biopsy with final diagnosis of MRI+/US- lesions.

\begin{tabular}{lcc}
\hline & \multicolumn{2}{c}{ Final diagnosis } \\
\cline { 2 - 3 } & Malignant & Benign \\
\hline CEUS alone & 11 & \\
Detected & 0 & 12 \\
$\quad$ Undetected & & 19 \\
CEUS plus needle biopsy & 10 & 0 \\
Malignant & 1 & 31 \\
Benign & & \\
\hline
\end{tabular}

CEUS: Contrast-enhanced ultrasonography.

sensitivity, and specificity were $71 \%$ (30/42), $100 \%$ (11/11), and 61\% (19/31), respectively, and, for CEUS plus needle biopsy, they were $98 \%(41 / 42), 91 \%(10 / 11)$, and $100 \%(31 / 31)$, respectively.

\section{Discussion}

The present study retrospectively investigated the diagnostic utility of third-look CEUS and CEUS-guided needle biopsy for MRI+/US- lesions. As a result, CEUS identified 55\% (23 in 42) of the MRI+/US- lesions, and CEUS-guided VAB/FNAC diagnosed $43 \%$ of them (10 in 23) as breast cancers. In addition, of these 10 malignant lesions, three (30\%) were invasive diseases, consistent with the Tozaki et al . report that MRI-guided needle biopsy for 102 MRI+/USlesions revealed 34 breast cancers (33\%) in which 6 lesions 


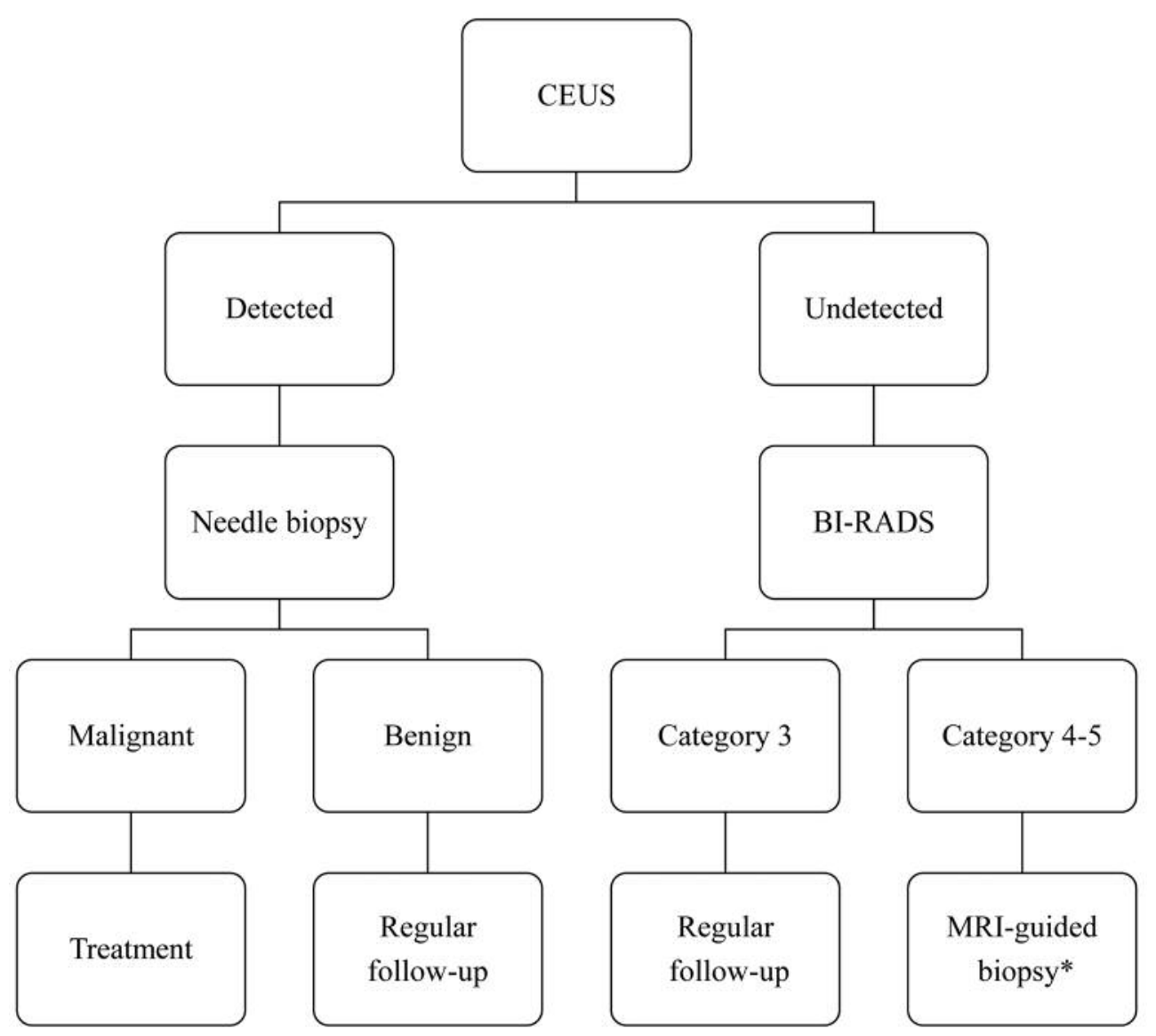

Figure 3: Diagnostic decision tree for the current management of MRI+/US- lesions. A decision tree for the current management of MRI+/USlesion is proposed. For MRI+/US-lesions, third-look CEUS is preferred to MRI-guided biopsy as the initial diagnostic procedure. If CEUS detects an MRI+/US- lesion, CEUS-guided needle biopsy should be performed. For MRI+/US-lesions determined to be malignant by means of CEUSguided biopsy, treatment for breast cancer is provided. For MRI+/US-lesions determined to be benign by means of CEUS-guided biopsy, regular follow-up is recommended. If the third-look CEUS cannot detect category 4/5 MRI+/US-lesions, MRI-guided needle biopsy should be considered. Regular follow-up can be offered for BI-RADS category 3 lesions undetectable by third-look CEUS. *The possibility of omission of MRI-guided biopsy for category 4 MRI+/US- lesions being undetected by third-look CEUS should be investigated in a future study. CEUS: Contrast-enhanced ultrasonography; BI-RADS: Breast Imaging Reporting and Data System; MRI: magnetic resonance imaging.

$(18 \%)$ were invasive cancers (16). The presence of invasive cancer with a significant frequency in the MRI+/US- lesions seemed to indicate a clinical importance of biopsy of such lesions as well as of development of a more convenient method than MRI-guided biopsy such as the CEUS-guided biopsy presented in this study. In addition, in cohort 1 , each MRI+/US- lesion that CEUS-guided VAB/FNAB determined to be malignant was located in a different quadrant of the ipsilateral breast from the index tumor, or in the contralateral breast. This result indicates that CEUS followed by needle biopsy contributes to the decision making of operation methods for breast cancer.

In recent years, Nykanen et al. first reported that CEUS detected $50 \%$ of a limited number of MRI+/US- breast lesions $(n=10)$ and that CEUS-guided needle biopsy or surgical resection diagnosed $80 \%$ of them as malignant (11). The rate of malignancy diagnosed by CEUS-guided biopsy was higher in the Nykanen study than in our study, whereas the identification rate of MRI+/US- lesions was similar between the two studies. This difference in the rate of malignancy may be explained by the fact that their study included only malignant or suspicious (i.e., BI-RADS category 4-5) MRI lesions, whereas our study included BIRADS category 3 lesions as well as BI-RADS category 4 and 5 lesions. Indeed, in the present study, BI-RADS category 4-5 lesions showed a significantly higher rate of malignancy $(42 \%, 10$ in 24$)$ than BI-RADS category 3 lesions $(6 \%, 1$ in 18$)(p<0.05)$. 
Of the patients whose MRI+/US- lesions were diagnosed as benign by CEUS-guided VAB/FNAC $(n=13)$, one patient underwent additional microdochectomy for the diagnosis of a BI-RADS category 3 lesion, because bloody nipple discharge continued, which may indicate the possibility of a sampling error, and the lesion was finally diagnosed as DCIS. However, all of the remaining 12 MRI+/US- lesions were followed up with no clinical evidence of malignancy. Of the 19 MRI+/US- lesions undetectable by third-look CEUS, nine lesions that were resected and pathologically evaluated, showed no abnormal findings. In addition, the remaining 10 MRI+/US- lesions were followed up with no evidence of clinical malignancy.

Our results demonstrated that CEUS followed by needle biopsy could achieve a high sensitivity $(91 \%)$ and specificity $(100 \%)$ in the diagnosis of MRI+/US- lesions, and even CEUS alone could achieve a high sensitivity (100\%) with a lower specificity $(61 \%)$. It is important that none of the CEUS-undetected, MRI+/US- lesions (BI-RADS category 3/4) were found to be malignant, suggesting that such lesions can be followed up without MRI-guided biopsy. Thus, the third-look CEUS for MRI+/US- lesions may appear to contribute to a reduction of the number of MRI-guided biopsies, especially in BI-RADS categories 3-4 MRI+/USlesions even though the number of patients included in the present study is limited.

Based on the results of the present study, a proposal of the decision tree for the current management of MRI+/US- lesions is shown in Figure 3. For MRI+/USlesions, third-look CEUS is preferred to MRI-guided biopsy as an initial diagnostic procedure. When CEUS detects MRI+/US- lesions, CEUS-guided needle biopsy should be performed. For MRI+/US- lesions that CEUSguided biopsy found to be benign, regular follow-up is recommended. If third-look CEUS cannot detect category 4/5 MRI+/US- lesions, MRI-guided needle biopsy should be considered, due to a high probability that the lesions are malignant. The possibility of omission of MRI-guided biopsy for category $4 \mathrm{MRI}+\mathrm{US}$ - lesions being undetected by third-look CEUS should be investigated in a future study. Regular follow-up can be offered for asymptomatic BI-RADS category 3 lesions undetectable by third-look CEUS.

In conclusion, our results suggest that third-look CEUS and CEUS-guided VAB/FNAC are useful diagnostic tools for breast MRI+/US- lesions and are more easily available in most hospitals than MRI-guided VAB. However, CEUSguided needle biopsy cannot replace the MRI-guided needle biopsy completely, because CEUS could not detect all the MRI+/US- lesions. We propose a decision tree for the current management of MRI+/US- lesions, including CEUS followed by CEUS-guided needle biopsy as an initial diagnostic procedure, which may contribute to decreasing the number of MRI-guided biopsies. To confirm our present findings on the value of CEUS plus needle biopsy, further study, including more patients with a longer follow-up period, is needed.

\section{Conflicts of Interest}

Dr. Noguchi has received research funding and honoraria from Daiichi-Sankyo Co., Ltd., for work performed outside of the current study. Dr. Shimazu has received honoraria from Daiichi-Sankyo Co., Ltd., for work performed outside of the current study.

\section{Authors' Contributions}

TM, SK and SN designed the study, and wrote the initial draft of the manuscript. All Authors have contributed to data collection, analysis and interpretation, and critically reviewed the manuscript.

\section{Acknowledgements}

The Authors are grateful to Yukiko Tokuda, MD, of the Department of Diagnostic and Interventional Radiology at Osaka University Hospital, for her contribution to radiological diagnosis. This research did not receive any specific grant from funding agencies in the public, commercial, or not-for-profit sectors.

\section{References}

1 Yi A, Cho N, Yang KS, Han W, Noh DY and Moon WK: Breast cancer recurrence in patients with newly diagnosed breast cancer without and with preoperative MR imaging: A matched cohort study. Radiology 276(3): 695-705, 2015. PMID: 25915100, DOI: 10.1148/radiol.2015142101

2 Hill MV, Beeman JL, Jhala K, Holubar SD, Rosenkranz KM and Barth RJ Jr.: Relationship of breast MRI to recurrence rates in patients undergoing breast-conservation treatment. Breast Cancer Res Treat 163(3): 615-622, 2017. PMID: 28315967, DOI: 10.1007/s10549-017-4205-9

3 Houssami N, Turner R, Macaskill P, Turnbull LW, McCready DR, Tuttle TM, Vapiwala N and Solin LJ: An individual person data meta-analysis of preoperative magnetic resonance imaging and breast cancer recurrence. J Clin Oncol 32(5): 392-401, 2014. PMID: 24395846, DOI: 10.1200/ JCO.2013.52.7515

4 Peters NH, Borel Rinkes IH, Zuithoff NP, Mali WP, Moons KG and Peeters PH: Meta-analysis of MR imaging in the diagnosis of breast lesions. Radiology 246(1): 116-124, 2008. PMID: 18024435, DOI: 10.1148/radiol.2461061298

5 Spick C and Baltzer PA: Diagnostic utility of second-look us for breast lesions identified at $\mathrm{mr}$ imaging: Systematic review and meta-analysis. Radiology 273(2): 401-409, 2014. PMID: 25119022, DOI: 10.1148/radiol.14140474

6 Weinstein SP, Hanna LG, Gatsonis C, Schnall MD, Rosen MA and Lehman CD: Frequency of malignancy seen in probably benign lesions at contrast-enhanced breast $\mathrm{mr}$ imaging: Findings from acrin 6667. Radiology 255(3): 731-737, 2010. PMID: 20501712, DOI: $10.1148 /$ radiol.10081712 
7 Imschweiler T, Haueisen H, Kampmann G, Rageth L, Seifert B Rageth C, Freiwald B and Kubik-Huch RA: MRI-guided vacuum-assisted breast biopsy: Comparison with stereotactically guided and ultrasound-guided techniques. Eur Radiol 24(1): 128135, 2014. PMID: 23979106, DOI: 10.1007/s00330-013-2989-5

8 Han BK, Schnall MD, Orel SG and Rosen M: Outcome of MRIguided breast biopsy. AJR Am J Roentgenol 191(6): 1798-1804, 2008. PMID: 19020252, DOI: 10.2214/AJR.07.2827

9 Mann RM, Kuhl CK, Kinkel K and Boetes C: Breast MRI: Guidelines from the european society of breast imaging. Eur Radiol 18(7): 1307-1318, 2008. PMID: 18389253, DOI: 10.1007/s00330-008-0863-7

10 Uematsu T, Takahashi K, Nishimura S, Watanabe J, Yamasaki S, Sugino T, Oishi T, Kakuda Y, Sato M and Hayashi T: Real-time virtual sonography examination and biopsy for suspicious breast lesions identified on MRI alone. Eur Radiol 26(4): 1064-1072, 2016. PMID: 26135000, DOI: 10.1007/s00330-015-3892-z

11 Nykanen A, Arponen O, Sutela A, Vanninen R and Sudah M: Is there a role for contrast-enhanced ultrasound in the detection and biopsy of MRI only visible breast lesions? Radiol Oncol 51(4): 386-392, 2017. PMID: 29333116, DOI: 10.1515/raon-2017-0049

12 Miyamoto Y, Ito T, Takada E, Omoto K, Hirai T and Moriyasu F: Efficacy of sonazoid (perflubutane) for contrast-enhanced ultrasound in the differentiation of focal breast lesions: Phase 3 multicenter clinical trial. AJR Am J Roentgenol 202(4): W400407, 2014. PMID: 24660739, DOI: 10.2214/AJR.12.10518
13 Tardivon AA, Athanasiou A, Thibault F and El Khoury C: Breast imaging and reporting data system (birads): Magnetic resonance imaging. Eur J Radiol 61(2): 212-215, 2007. PMID: 17156958, DOI: $10.1016 /$ j.ejrad.2006.08.037

14 Shimazu K, Ito T, Uji K, Miyake T, Aono T, Motomura K, Naoi Y, Shimomura A, Shimoda M, Kagara N, Kim SJ and Noguchi $\mathrm{S}$ : Identification of sentinel lymph nodes by contrast-enhanced ultrasonography with sonazoid in patients with breast cancer: A feasibility study in three hospitals. Cancer Med 6(8): 1915-1922, 2017. PMID: 28766883, DOI: 10.1002/cam4.1142

15 Conant EF, Beaber EF, Sprague BL, Herschorn SD, Weaver DL, Onega T, Tosteson AN, McCarthy AM, Poplack SP, Haas JS, Armstrong K, Schnall MD and Barlow WE: Breast cancer screening using tomosynthesis in combination with digital mammography compared to digital mammography alone: A cohort study within the prospr consortium. Breast Cancer Res Treat 156(1): 109-116, 2016. PMID: 26931450, DOI: 10.1007/s10549-016-3695-1

16 Tozaki M, Yamashiro N, Sakamoto M, Sakamoto N, Mizuuchi N and Fukuma E: Magnetic resonance-guided vacuum-assisted breast biopsy: Results in 100 japanese women. Jpn J Radiol 28(7): 527533, 2010. PMID: 20799018, DOI: 10.1007/s11604-010-0464-7

Received December 19, 2018

Revised January 12, 2019

Accepted January 14, 2019 